\section{Dr. Ishizaka, et al reply}

\section{To the Editor:}

We thank Dr. Harris for his comments on our study, in which we analyzed the relationship between changes in obesity measures and those in serum uric acid (UA) levels in individuals who underwent general health screening 2 years running and were free from antihyperuricemic medication ${ }^{1}$. Serum UA is reported to be higher in Japanese individuals with general and abdominal obesity ${ }^{2-4}$. In addition, several studies have shown that weight change may lead to a change in serum UA levels ${ }^{5,6}$; however, few data are available on the influence of change in waist circumference on serum UA levels in Japanese individuals.

We found that there was a significant association between the percentage change in BMI (\%dBMI) and the percentage change in serum UA (\%dUA) in men and postmenopausal women. On the other hand, change in waist circumference was not associated with \%dUA independently of $\%$ dBMI. These data suggest that reducing BMI may be a tool for reduction of serum UA levels in men and postmenopausal women. As Dr. Harris pointed out, however, the correlation coefficients of \%dBMI for \%dUA were, although statistically significant, relatively small, indicating that reduction of body weight alone may have only a minor effect on controlling serum UA levels.

We do not insist that antihyperuricemic medication would be unnecessary for the treatment of hyperuricemia when weight reduction is successful. Our data may, however, provide the limitations, as well as the effectiveness, of weight/waist circumference reduction in terms of serum UA reduction. Our data may also provide an estimation of the extent of serum UA level changes with BMI or waist circumference during a 1-year period. Such information is useful considering that the potential modification of life habits that could lead to an improvement in metabolic profile is an important step in the management of hyperuricemia and gout in the guidelines of the Japanese Society of Gout and Nucleic Acid Metabolism. We agree with Dr. Harris that, in subjects with serum UA levels $\geq 8.0 \mathrm{mg} / \mathrm{dl}$, normalization of serum UA levels only by reducing body weight may be difficult and/or impractical. We calculated the percentage change in UA during a 1-year interval in our study ${ }^{1}$; therefore, the effect of weight and waist circumference reduction on change in UA over longer periods also needs to be studied.
NOBUKAZU ISHIZAKA, MD, Department of Cardiovascular Medicine, University of Tokyo Graduate School of Medicine; YUKO ISHIZAKA, MD; AKIKO TODA, MD; MIZUKI TANI, MD, Center for Multiphasic Health Testing and Services, Mitsui Memorial Hospital; KAZUHIKO KOIKE, MD, Department of Gastroenterology, University of Tokyo Graduate School of Medicine; MINORU YAMAKADO, MD, Center for Multiphasic Health Testing and Services, Mitsui Memorial Hospital; RYOZO NAGAI, MD, Department of Cardiovascular Medicine, and Gastroenterology, University of Tokyo Graduate School of Medicine, Hongo 7-3-1 Bunkyo-ku, Tokyo 113-8655, Japan. Address correspondence to Dr. N. Ishizaka, Department of Cardiovascular Medicine, University of Tokyo Graduate School of Medicine;

E-mail: nobuishizka-tky@umin.ac.jp

\section{REFERENCES}

1. Ishizaka N, Ishizaka Y, Toda A, Tani M, Koike K, Yamakado M, et al. Changes in waist circumference and body mass index in relation to changes in serum uric acid in Japanese individuals. J Rheumatol 2010;37:410-6.

2. Takahashi S, Yamamoto T, Tsutsumi Z, Moriwaki Y, Yamakita J, Higashino K. Close correlation between visceral fat accumulation and uric acid metabolism in healthy men. Metabolism 1997;46:1162-5.

3. Matsuura F, Yamashita S, Nakamura T, Nishida M, Nozaki S, Funahashi T, et al. Effect of visceral fat accumulation on uric acid metabolism in male obese subjects: visceral fat obesity is linked more closely to overproduction of uric acid than subcutaneous fat obesity. Metabolism 1998;47:929-33.

4. Kawada T, Otsuka T, Katsumata M, Suzuki H. Serum uric acid is significantly related to the components of the metabolic syndrome in Japanese working men. J Cardiometab Syndr 2007;2:158-62.

5. Borkan GA, Sparrow D, Wisniewski C, Vokonas PS. Body weight and coronary disease risk: patterns of risk factor change associated with long-term weight change. The Normative Aging Study. Am J Epidemiol 1986;124:410-9.

6. Nakanishi N, Nakamura K, Suzuki K, Matsuo Y, Tatara K. Relation of body weight change to changes in atherogenic traits; a study of middle-aged Japanese obese male office workers. Ind Health 2000;38:233-8.

J Rheumatol 2010;37:5; doi:10.3899/jrheum.100071 